\title{
Sex Differences in Mechanisms and Outcome of Neonatal Hypoxia-Ischemia in Rodent Models: Implications for Sex-Specific Neuroprotection in Clinical Neonatal Practice
}

\author{
Courtney A. Hill and R. Holly Fitch \\ Department of Psychology, University of Connecticut, 406 Babbidge Road, Storrs, CT 06269, USA \\ Correspondence should be addressed to Courtney A. Hill, cahill@wihri.org
}

Received 7 September 2011; Revised 3 November 2011; Accepted 16 November 2011

Academic Editor: Robin L. Haynes

Copyright (C) 2012 C. A. Hill and R. H. Fitch. This is an open access article distributed under the Creative Commons Attribution License, which permits unrestricted use, distribution, and reproduction in any medium, provided the original work is properly cited.

\begin{abstract}
Clinical findings show that male infants with hypoxic-ischemic injury (HI) fare more poorly than matched females on cognitive outcomes. Rodent models of neonatal hypoxia-ischemia support this difference, with data showing that perinatal brain injury leads to long-term behavioral deficits primarily in male rodents and in female rodents treated with early androgens. Results support the idea that sex-specific gonadal hormones may modulate developmental response to injury and dovetail with overwhelming evidence of developmental androgen effects on typical brain morphology and behavior. However, mechanisms underlying sex differences in response to early brain injury may be more complicated. Specifically, activation of cell death pathways in response to HI may also differ by sex. In females, the preferential activation of the caspase-dependent apoptotic pathway may actually afford greater protection, potentially due to the actions of X-linked inhibitor of apoptosis (XIAP) within this pathway. This contrasts the pattern of preferential activation of the caspase-independent pathway in males. While an integrated model of sex-specific hormonal and genetic modulation of response to early injury remains to be fully elucidated, these findings suggest that infants might benefit from sex-specific neuroprotection following HI injury.
\end{abstract}

\section{Introduction}

Perinatal hypoxic-ischemic injury (HI; concurrent oxygen/blood deprivation in the brain) represents a major cause of mortality and long-term neurologic morbidity in premature/very low-birth-weight (VLBW) infants $(<1500 \mathrm{~g})$ and in term infants suffering birth trauma [1]. In premature infants, the vulnerability of the underdeveloped neural vascular system, coupled with poor cerebral autoregulation [2], can often result in intraventricular or periventricular hemorrhagic injury (IVH-PVH; bleeding within or surrounding the ventricles [3]). These bleeds, primarily located in the subependymal germinal matrix, lead to some immediate cell necrosis as well as a progressive apoptotic cell death cascade of germinal matrix and glial precursor cells [3]. In addition, poor cerebral autoregulation (including reperfusion failure) in preterm infants can lead to periventricular leukomalacia (PVL), a nonhemorrhagic ischemic injury associated with loss of white matter surrounding the ventricles [4]. Moreover, underdevelopment of the lungs can lead to reduced oxygenation of the blood, thus resulting in hypoxic conditions within the brain of premature infants. Animal models of acute preterm HI injury include the Rice-Vannucci model of unilateral carotid artery ligation followed by a period of exposure to $8 \%$ oxygen prior to postnatal day 7 (P7), typically performed in rodents [5-7]. Additional models are also used in which fetal blood supply is diminished by clamping of the placental blood supply $[8,9]$, and/or by raising dams in a low-oxygen environment for a period of days $[10,11]$.

In term infants, HI injury typically results from complications of birth (e.g., cord compression, placental disruption/failure, or cord asphyxia [1, 7, 12-14]) and can result in cerebral white matter injury typical of cerebral palsy [15] or in gray matter injury $[1,16,17]$. Thus, term-born children experiencing asphyxia exhibit injuries 
more typical of hypoxic-ischemic encephalopathy (HIE), in contrast to injuries exhibited by preterm children (i.e., IVHPVH, PVL). Animal models of term injury include the RiceVannucci method performed on P7-P10 [5-7] or methods of middle cerebral artery occlusion (MCAO, [18]). Although the mechanisms of neural damage in preterm versus term populations differ, these varied forms of injury result in similar activation of acute necrotic and delayed apoptotic cell death mechanisms that impact on cell populations most vulnerable during the time of injury [5-7].

Not surprisingly, the long-term consequences of neonatal $\mathrm{HI}$ injury in both populations can be severe. Nearly $50 \%$ of term-born infants suffering severe HIE die within weeks of birth, while up to $25 \%$ of those surviving exhibit permanent neuropsychological dysfunction [7]. Similarly, a $50 \%$ mortality rate exists for preterm infants experiencing severe HI, with $80 \%$ of survivors experiencing long-term complications [19], including reductions in cerebellar [20], cortical, and hippocampal volumes [21] associated in turn with cognitive and behavioral deficits, deficits in verbal and language domains [22, 23], reduced IQ measures [24], cerebral palsy, and mental retardation [25]. What is surprising, however, is the disproportionate incidence of, and increased severity of effects following, neonatal HI injury in males. Not only are male infants more vulnerable to perinatal insult (showing higher incidence of IVH and increased rates of mortality from prematurity or stillbirth), they also suffer more long-term cognitive deficits as compared to females with comparable injury $[23,25-31]$. In fact, males in general show increased risk for brain-based developmental disorders, including speech and language disorders, stutter, dyslexia, autism, learning disabilities, attention-deficit-hyperactivity disorder, and cerebral palsy as compared to females [26, $27,30,32]$. Importantly, males suffering intracranial bleeds at birth also display significantly lower full-scale, verbal, and performance IQ at early school age as compared to females matched for degree of prematurity and severity of intracranial bleed [33]. Overall, evidence suggests that among infants at risk for $\mathrm{HI}$, females may be at a quite significant advantage as compared to their male counterparts who are two times more likely to experience prenatal anoxia, hemorrhage, and infection, and 1.8 times more likely to suffer cerebral birth trauma [26-28, 30].

Despite the overwhelming evidence of sex differences in outcome following neonatal HI injury, many researchers appear to remain naïve to the importance of sex in perinatal injury models and continue to utilize only male animals in research studies. However, recent work concerning hormones present during the perinatal period, as well as sex differences in mechanisms of cell death, have begun to illustrate dynamic and differing processes occurring in the neonatal brain following injury and emphasize the need for studies to include both sexes. This work suggests, first, that the substantially elevated level of testosterone present in human male fetuses during gestation through the first year of life [34-36] may enhance neuronal excitotoxicity following hypoxic-ischemic insult $[37,38]$ and may contribute to exacerbated deficits in males [39, 40]. Second, evidence suggests that following such injury, male and female cells diverge in the proportional activation of caspase-dependent and caspase-independent pathways leading to apoptotic death [41-43]. In fact, this difference may contribute to outcomes that show males to be more vulnerable to early brain damage [44-46]. Finally, data indicate that females may possess a gene-linked advantage through a family of inhibitors of apoptosis (IAPs [47]), the most potent being Xlinked IAP (XIAP [48]). XIAP is known to act on the caspasedependent apoptotic pathway [48-51], and it is possible that increased expression of XIAP [52] in females may contribute to a female advantage following neonatal HI. Taken together, this evidence suggests an interplay of hormonal modulation and genetically determined apoptotic mechanisms, through which perinatal females may be afforded a level of protection against HI injury that is greater than for perinatal males. The current paper will focus on factors that may play key roles in the outcome of hypoxic-ischemic events experienced by males and females, including perinatal exposure to sexspecific gonadal hormones and sex-specific cell death mechanisms. Research in this area could lead to the discovery and clinical implementation of sex-specific neuroprotectants for infants suffering from HI injury.

\section{Early Hormonal Factors}

Sex differences in androgen levels represent one principal difference in the male versus female neonatal brain and lead to substantial effects on brain morphology and subsequent behavior [34-36, 53-59]. Human testes develop around gestational week $(\mathrm{GW}) 6$, with testosterone from the testesas well as from the fetal adrenals (as a by-product of corticosteroid production)_circulating at detectable plasma levels in males by GW $8[34,35]$. Testosterone secretion, however, is highest from GW 10 to 20, falling to lower levels by GW 24, followed by a second transient testosterone surge on the day of birth (in response to the drop in placental estrogen). In humans, testosterone levels gradually increase during the first week of life and remain high for the first year, peaking during the 3rd-4th month at levels similar to the second stage of puberty (200-300 ng/dL; [35]). Through aromatization, testosterone can be converted to 17$\mathrm{B}$ estradiol, thus allowing it to bind to estrogen receptors within the brain $[53,54,60]$. In fetal male rats, where plasma testosterone is significantly higher than female littermates beginning at embryonic day 18 (E18) through P5 [61], the conversion of circulating testosterone to estradiol results in neural and behavioral masculinizing effects [36, 53]. However, no studies of which we are aware directly support this mechanism in humans. Difficulties in ascertaining the role of aromatization in human sexual differentiation reflect experimental constraints [53], but some evidence does support a role for aromatization in human development [62].

Human female fetuses are also exposed to androgens from the fetal adrenal glands, as well as the maternal adrenals, ovaries, and fat-though the amount is insufficient for masculinization. In humans, it is believed that a negative feedback loop between the fetal adrenal cortex and the 
anterior pituitary corticotrophins minimizes female adrenal androgen secretion, acting as a transient mechanism that safeguards early human female development from virilization [63]. The fetal ovaries also develop at approximately GW 7, though no circulatory estrogen of fetal ovarian origin is present until very late in gestation $[34,35]$. Likewise, studies of ovarian secretion of estrogen in the rat reveal detectable levels 5 days after birth (corresponding to the late third trimester human; [56]), although some central steroidogenesis may occur [64]. In rodents, females circumvent masculine development via maternal estrogen (consistent with masculinization via intracellular conversion of androgens as discussed above) through alpha-fetoprotein, a binding globulin found in late-gestation fetuses. This protein binds to estrogen within the bloodstream thereby rendering it inactive and preventing virilization of the female rodent brain $[36,54]$.

2.1. Testosterone and Brain Injury. As noted above, the presence of testosterone during development represents one of the foremost differences between neonatal male and female brains. However, despite a large and dynamic literature concerning modulatory effects of gonadal hormones on pathologic and behavioral response to stroke injury in adults, research data concerning hormonal modulation of injury in neonates remains scant (but see [65]). A brief review of this adult literature illustrates that studies using an induced injury model simulating adult stroke-middle cerebral artery occlusion (MCAO) - show consistent evidence that males benefit after injury from acute testosterone depletion, while the presence of testosterone increases glutamate toxicity following injury [37]. Furthermore, testosterone and its metabolite dihydrotestosterone (DHT) have been shown to increase stroke damage in young adult rats $[37,66,67]$, while testosterone concentration was found to be inversely associated with stroke severity and 6-month mortality [68, 69]. Interestingly, young male rats were also found to incur larger strokes than their older counterparts, an effect hypothesized to be due to the ability of testosterone to alter the susceptibility of the brain to ischemic damage in an age-dependent manner. Alternately, though aromatase levels are stable over age (and thus the protection afforded to aged males is not likely due to increased capability for aromatization of testosterone to estrogen [70]), the declining effects of stroke damage in older males may reflect higher levels of testosterone in young rats [70].

In neonatal animals, baseline sex differences have been seen with an early hypoxia model $[10,71]$ as well as an HI model $[40,72,73]$ of brain injury - both of which have shown that males exhibit increased brain volume loss [10,40, $71,74]$, disrupted myelination [10], and increased behavioral deficits [40,71-73] following injury as compared to liketreated females. As with adult injury models, there is also some evidence that the presence of androgens can exacerbate induced brain damage-for example, following GABA-A mediated excitotoxicity [65]. Other studies of a rat model of focal ischemic injury leading to developmental cortical malformation (microgyria) found that androgenizing female rat pups via testosterone propionate (TP) prior to and following induction of microgyria via focal cortical freezing lesion on P1 led to a developmental shift in medial geniculate nucleus (MGN) neuronal size distribution in adulthoodsimilar to that seen in male microgyric rats-while vehicletreated microgyric females were found to be identical to sham females (and showed no disruption of the MGN [39]). With regard to the specific long-term effects of testosterone as a modulator of neonatal $\mathrm{HI}$ injury, we are aware of only one study performed to date [40]. In this study, vehicletreated male and female rat pups, as well as female rat pups that had been treated on postnatal days $1-5$ (P1-5) with superphysiologic levels of testosterone propionate (TP), received the Rice-Vannucci HI procedure [5] on P7. Results showed subsequent deficits in auditory processing ability in males and TP-treated females with induced neonatal HI, while no effect of HI was found in vehicle-treated females [40], thus demonstrating the apparent deleterious effects of androgen exposure in modulating behavioral deficits associated with HI.

From these cumulative studies, it is evident that testosterone acts in some manner to exacerbate the response to early hypoxic-ischemic injury in rats, though the specific mechanism(s) of action remain to be defined. Moreover, since an aromatizable form of testosterone propionate was used in the above study [40], it is not possible to determine whether these effects were modulated directly by androgens or via intracellular conversion to estrogen. Future studies looking at neonatal $\mathrm{HI}$ while manipulating testosterone receptors, estrogen receptors, and/or aromatase blockers could potentially dissociate or clarify this issue. Nonetheless, further research in the area of neonatal testosterone exposure may help to better characterize the protection afforded to females, which potentially could be adapted to males through some form of neuroprotective treatment. Although the generalized use of androgen-blocking manipulations in male infants would be a clinically untenable intervention, a viable option could be to identify the delineated mechanisms of androgenic exacerbation of injury and block those specific effects only.

2.2. Estrogen and Brain Injury. It must also be noted that evidence from animal models of adult stroke shows substantial beneficial and protective effects of estrogen modulation [75-77]. In fact, adult female animals have a lower incidence of naturally occurring stroke [78] and show less sensitivity than male animals to the damaging effects of focal or global ischemic injury [79], and-in strains displaying conditions known to be stroke risk factors in humans (i.e., hypertension)-female animals display less tissue damage than males following induced stroke [80]. This female advantage has been attributed at least in part to protective effects of ovarian steroid hormones, since interventions that reduce estrogens (i.e., ovariectomy, estrogen receptor blockade, and natural aging) have all reduced differences in stroke outcome between the sexes [75]. Further, induced stroke during metestrus (when estrogen is lowest) increases tissue damage in comparison to strokes occurring during proestrus (when estrogen is highest; [77]). It should be noted that the effects of estrogen on adult human stroke 
are less clear, and many of the successful studies of estrogen replacement in animal models have failed to translate to human clinical populations $[75,76]$. Nonetheless, whatever the protective mechanism(s) of estrogen may be, they are less likely to fully account for the neonatal effects described here, since female protection has been shown in animal models of neonatal brain injury, when minimal circulating estrogen from the quiescent neonatal ovaries is present $[40,68,72,73$, 81,82 ] although central steroidogenesis may occur [64]. Still, it is certainly possible that some late developmental beneficial effects of ovarian estrogen on neural reorganization after injury could occur.

These hormonal data, taken together, suggest that early androgen exposure in males may be a primary contributor to the modulation of sex differences in response to HI injury (although whether these effects occur through aromatization cannot be determined, based on data collected to date). However, another promising line of research aimed at exploring sex differences in response to brain injury has begun to examine possible sex differences in the mechanisms of cell death following injury. These findings have led researchers to believe that hormonal differences are not the only key factor modulating sex differences in response to injury, and that the apoptotic cascade may be differentially activated in the male and female brain following $\mathrm{HI}$.

\section{The Apoptotic Cascade}

Apoptosis, or programmed cell death, can be triggered by various events including DNA damage, cytotoxic drugs, a lack of survival signals, and developmental death signalsamong a variety of other mechanisms [83]. With regard to neuronal death in response to hypoxia-ischemia, events are initially triggered by a deprivation of oxygen and glucose supply to the cell(s), which depresses both adenosine triphosphate (ATP) synthesis as well as the cellular uptake of glutamate. The accumulation of excess extracellular glutamate triggers an increase in glutamate receptor (NMDA, AMPA, and kainate) activation and prolonged depolarization, leading to increased calcium and sodium influx. Sodium influx through AMPA and kainate receptors leads to cell swelling and rapid necrotic cell death, while calcium influx through NMDA and AMPA receptors lacking the GluR2 subunit (rendering the channel open to calcium) activates neuronal nitric oxide synthase (nNos). nNos, in turn, leads to the production of the free radicals, nitric oxide (NO), and peroxynitrate (ONOO). In caspase-independent-mediated cell death, a reduction of nicotinamide adenine dinucleotide $\left(\mathrm{NAD}^{+}\right.$, a high energy molecule) is caused by activation of poly(ADP-ribose) polymerase-1 (Parp-1, a DNA repair enzyme), leading to release of apoptosis-inducing factor (AIF) and endonuclease G from the mitochondria to the nucleus of the cell, and ultimately cell death [49-51]. Through a second caspase-dependent pathway, the increase in nNos ultimately leads to mitochondrial dysfunction and the translocation of cytochrome-c from the mitochondria to the nucleus, signaling apoptotic protease-activating factor1 (APAF-1) and the formation of the apoptosome. The

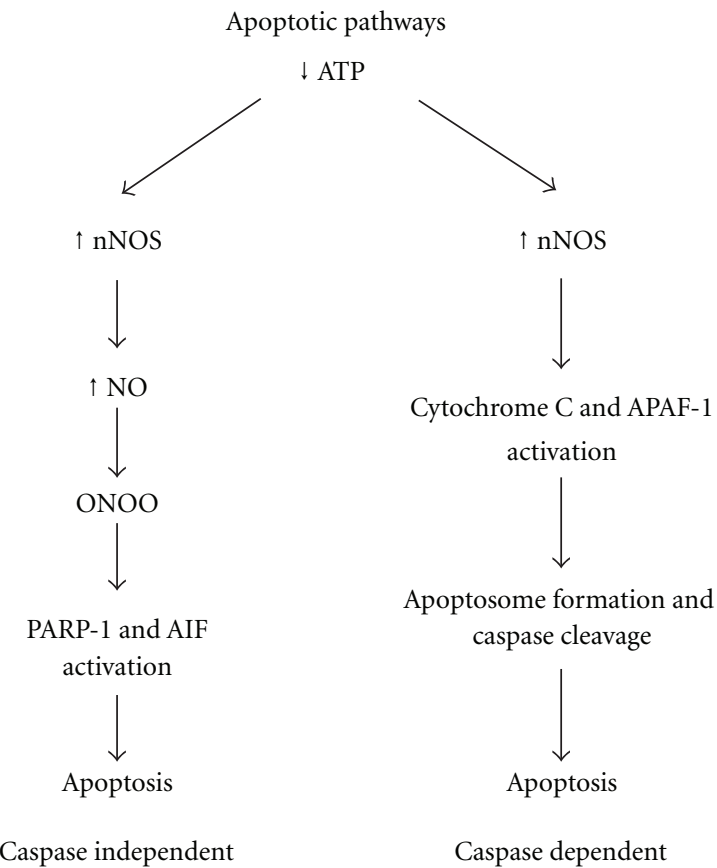

FIGURE 1: A diagram of the progression of caspase-independent and -dependent apoptotic mechanisms.

apoptosome binds with caspase-9 (the initiator caspase), which in turn cleaves downstream caspases 3, 6, and 7 (effector caspases) causing chromatin condensation, DNA fragmentation, and ultimately cell death [42, 50, 83-85], (see Figure 1).

Interestingly, research has revealed the sexes to differentially favor one of these two pathways (though not exclusively), with females relying more heavily on the caspasedependent pathway and males largely utilizing the caspaseindependent pathway of cell death following HI insult. These data derive from injury models in both adult stroke (MCAO) and neonatal HI models [41-46, 80, 86, 87].

3.1. Apoptotic Cascades and Brain Injury. In adult stroke models, male and female Parp-1-deficient mice both display a reduction in Parp-1 and AIF production, suggesting the activation of the caspase-independent apoptotic pathway by both sexes. However, only male Parp-1-deficient animals exhibited a reduction in stroke-induced brain damage following MCAO [86]. Likewise, female (but not male) mice were largely resistant to endotoxin-induced mortality, and Parp-1 inhibition decreased endotoxin-induced vascular and inflammatory response in male (but not female) mice [88]. Interestingly, ovariectomy partially reversed the protection normally seen in females, suggesting a modulating role of estrogen [88]. Similarly, inhibition of Parp-1 and nNOS was found to protect male animals from the damaging effects of MCAO (but not females, [89]). In fact, Parp-1 inhibition increased stroke damage in intact females and estrogen-replaced ovariectomized females, again suggesting a mediating role of estrogen [89]. Though the results of these two studies suggest that ovarian hormones may play a role in 
modulating caspase-independent cell death in adult models, it is important to note that sex differences in neonatal HI are found when minimal circulating estrogen is present (though evidence indicates central steroidogenesis may be occurring [64]). These neonatal studies also report evidence of predominant male use of the caspase-independent pathway, suggesting such sex differences may not be exclusively mediated by exogenous hormones (see below). Likewise, preferential activation of the caspase-dependent apoptotic pathway has been seen in adult female animals following $\mathrm{MCAO}$, as increased cytochrome-c release and caspase3 cleavage was found relative to males, while inhibition of caspase activation was found to be neuroprotective in female animals only. This benefit was extended to ovariectomized and estrogen-replaced female animals, indicating these effects to be independent of hormones [80].

Recent work exploring cell death mechanisms in neonatal models also indicates sex differences in the preferred apoptotic pathway following early HI [41, 42]. With regard to the caspase-independent apoptotic pathway, both Parp1 and AIF have been found in higher concentration in the brains of male mice following $\mathrm{P} 9 \mathrm{HI}$ injury, as compared to the brains of HI-injured females [43]. Likewise, significant protection from P7 HI injury has been shown in Parp-1 knockout male mice, though no comparable protection was seen in females [44]. Other studies report that cytochrome$\mathrm{c}$ and various caspases-which are active in the caspasedependent pathway-have been found in higher concentration in female as compared to male mice following P9 $\mathrm{HI}$ injury [43]. Likewise, inhibition of caspase cleavage has been shown to be neuroprotective in female (but not male) animals following P3 or P7 HI $[45,46]$, where translocation of cytochrome-c was prevented. Finally, neuronal cultures (absent of circulating hormones) subjected to cytotoxic challenge showed differing pathways of cell death-with XY neurons predominantly utilizing the AIF-mediated caspaseindependent apoptotic pathway and XX neurons activating the cytochrome-c, caspase-dependent apoptotic pathway [38]. It should be noted that no studies (of which we are aware) have quantified the exact proportional activation of caspase-dependent and -independent apoptosis for either sex, but instead have measured the activation of elements specific to each pathway or the relative protection afforded to one sex over the other following knockout/inhibition of elements specific to one pathway as stated above. These sex differences are found to be significant in magnitude.

From these studies, it is evident that male and female neurons undergoing apoptosis capitalize on pathways that show sex differences unlikely to be exclusively related to hormones (though these apoptotic pathways are not exclusive to sex). However, detailed assessment of the potential interaction between the early (or concurrent) presence of gonadal hormones, and the activation of sex-specific apoptotic cascades, remains to be defined. Nonetheless, apoptosis is a major contributor to neuronal cell death and tissue loss following neonatal HI, and the development of neuroprotectants aimed at targeting the mechanisms most utilized by each sex represents a valuable venue of investigation for therapeutic interventions.

\section{A Gene-Linked Female Advantage}

An alternative or additional explanation for sex differences seen in neonatal HI outcome involves endogenous inhibitors of apoptosis. During development, the apoptotic cascade is a highly regulated process critical for healthy development and maintenance of tissue. This process of programmed cell death is counterbalanced by antiapoptotic signals that promote the survival of cells. A family of proteins, known as inhibitors of apoptosis (IAPs), serve as endogenous inhibitors of cell death $[47,90]$ and have been found to regulate apoptosis by blocking both the intrinsic and extrinsic mechanisms. Specifically, IAPs directly bind to and inhibit initiator and effector caspases $[47,49,51]$. The function of IAPs has recently been extended beyond its initial role in development and is now thought to play a role in processes such as cancer, tumor formation, autoimmune diseases, neurodegenerative disorders, and most recently, cell death following brain injury $[51,91]$.

Of the known IAPs, X-linked IAP (XIAP) is recognized to be the most potent [48]. XIAP effectively binds to the initiator caspase (caspase-9) and halts further cleavage of downstream caspases (caspases 3 and 7), thus preventing cell death [48-51]. XIAP has also been shown to bind and inhibit caspases 3 and 7 directly [48], and in vitro studies have revealed XIAP to severely inhibit nuclear destruction and cytochrome-c-induced caspase activation [48]. XIAP expression has been confirmed in both rodent and human brains following ischemic injury [92]. Moreover, it is understood that genetic balancing in females occurs via random inactivation of the second $\mathrm{X}$ chromosome; however, $15 \%$ of genes located on the second X chromosome always escape inactivation, and an additional 10\% sometimes escape inactivation [52]. Therefore, it is possible that females present with an increased expression of XIAP relative to males. And since XIAP acts specifically on the caspasedependent pathway of cell death preferentially activated in females, XIAP may play a role in the selective protection afforded to females following early $\mathrm{HI}$ injury.

4.1. X-Linked IAPs and Brain Injury. Currently very little is known about the regulation of IAPs and XIAP in neonatal $\mathrm{HI}$ injury, though surprisingly, results from studies of XIAP knockout [93] and overexpression [94] have largely failed to report sex differences in degree of tissue damage following early HI injury. Further investigation revealed that these results may be due to compensatory changes in other IAP family members (i.e., upregulation of c-IAP1 and c-IAP2 [95, 96]), with XIAP remaining a probable source of protection for females. One specific study examined the long-term behavioral effects of neonatal HI following inhibition of XIAP in male and female rats [73]. Based on cumulative evidence of sex differences in apoptotic mechanisms, coupled with evidence of potential female protection via XIAP, this study utilized embelin - a small molecule inhibitor of XIAP. Embelin binds to the BIR3 domain (the biding site of caspase-9) on the XIAP protein molecule [91], thus preventing endogenous inhibition of apoptosis by XIAP. Treatment 
with embelin increased neuropathological damage and lifelong behavioral deficits in $\mathrm{HI}$ females relative to vehicletreated $\mathrm{HI}$ females, while no comparable effects were seen in males [73]. Thus, results demonstrate both the reliance on specific pathways of cell death between the sexes, as well as the importance of XIAP in the protection afforded to females following injury.

Clearly more research is needed on the role of IAPs in hypoxic-ischemic neuronal death, but the studies presented here emphasize the need for an improved understanding of innate mechanisms of protection in male and female neonates. Future studies will be needed to assess the potential interaction of hormonal exposure and genetic differences in sex chromosome gene expression within brain cells, since sex differences in response to early injury are almost certainly influenced by a combination of these factors.

\section{Conclusions}

Neonatal HI is a major cause of infant mortality and long-term neurologic morbidity in both preterm and terminjured populations. It is evident that the consequences of neonatal $\mathrm{HI}$ injury are severe, yet the difference in outcome experienced between the sexes is surprising. Male infants not only exhibit increased risk for HI, but also display greater behavioral and cognitive disruption following $\mathrm{HI}$ injury as compared to matched female counterparts. Animal studies utilizing induced neonatal HI suggest that this sex discrepancy may be modulated by (1) the presence of sexspecific hormones (e.g., testosterone), (2) sex differences in the preferred mechanisms of apoptosis, and/or (3) the protective effect of IAPs (which may be in greater quantity in female brain) on the caspase-dependent apoptotic pathway. Indeed, all three of these mechanisms may interact with each other, and sex differences in the effects of neonatal HI outcome likely reflect an interplay of both genetic and hormonal factors. One possible study to dissociate these interactive mechanisms could entail the use of a fourcore genotype (FCG) mouse model (described in [97]), in which the Sry (testis determining) gene is deleted from the $\mathrm{Y}$ chromosome and inserted onto an autosome. A cross between this type of male and an XX female can then produce genetic females with insertion of the Y chromosome Sry gene modulating testicular development (thus leading to androgen exposure absent of all other $Y$ chromosome genes), and XY males with knockout of the Sry gene (who develop as phenotypic females). Exploration of the consequences of neonatal $\mathrm{HI}$ in mice with $\mathrm{Y}$ genes but no testosterone, and testosterone but no other $\mathrm{Y}$ genes, could allow a more indepth study of whether sex-based preference for apoptotic pathways may somehow be set by early androgen exposure, other genetic factors, or both.

In closing, further studies of the influence of both genetic and hormonal factors relevant to neonatal HI could have important clinical implications. For example, the modulation of hormonal mechanisms leading to increased damage in males, modulation of apoptotic cascades, or modulation of IAPs may all represent target candidates for therapeutic intervention in neonates suffering HI brain injury. Further, studies looking at neonatal HI while manipulating testosterone receptors, estrogen receptors, and/or aromatase blockers could potentially dissociate or clarify the mechanism of action promoting injury. Moreover, it seems plausible that a lack of exposure to placental hormones due to premature birth could also be detrimental to the neurological development of premature infants (though no studies, of which we are aware, have determined such effects). Given the tremendous amount of research focusing on sex differences in adult stroke, we suggest that future research should similarly focus on sex differences in the consequences of neonatal HI. In fact, research in this area could yield beneficial sex-specific neuroprotectants, with far reaching implications for improved clinical practice and treatment.

\section{Acknowledgments}

This research was supported by NIH Grant HD049792 and a grant from the University of Connecticut, Regional Campus Incentive Program (UCIG). Special thanks are due to Michelle Alexander for assistance with rodent surgeries and behavioral testing, as well as undergraduates Joseph Taitague and Vadim Kotlyar for additional help with histological assessment. Many thanks are also due to Chad Siegel for assistance in study planning and preparation and Dr. Louise McCullough for assistance in study planning and preparation, as well as editing input.

\section{References}

[1] A. Fatemi, M. A. Wilson, and M. V. Johnston, "Hypoxicischemic encephalopathy in the term infant," Clinics in Perinatology, vol. 36, no. 4, pp. 835-858, 2009.

[2] G. B. Boylan, K. Young, R. B. Panerai, J. M. Rennie, and D. H. Evans, "Dynamic cerebral autoregulation in sick newborn infants," Pediatric Research, vol. 48, no. 1, pp. 12-17, 2000.

[3] J. J. Volpe, "Hypoxic-ischemic encephalopathy and intracranial hemorrhage," in Neurology of the Newborn, WB Saunders, Philadelphia, Pa, USA, 2001.

[4] S. Takashima, M. Itoh, and A. Oka, "A history of our understanding of cerebral vascular development and pathogenesis of perinatal brain damage over the past 30 years," Seminars in Pediatric Neurology, vol. 16, no. 4, pp. 226-236, 2009.

[5] J. E. Rice III, R. C. Vannucci, and J. B. Brierley, "The influence of immaturity on hypoxic-ischemic brain damage in the rat," Annals of Neurology, vol. 9, no. 2, pp. 131-141, 1981.

[6] R. C. Vannucci and S. J. Vannucci, "Perinatal hypoxic-ischemic brain damage: evolution of an animal model," Developmental Neuroscience, vol. 27, no. 2-4, pp. 81-86, 2005.

[7] S. J. Vannucci and H. Hagberg, "Hypoxia-ischemia in the immature brain," Journal of Experimental Biology, vol. 207, part 18, pp. 3149-3154, 2004.

[8] F. K. Lotgering, J. M. Bishai, P. C. Struijk et al., "Absence of robust ischemic preconditioning by five 1-minute total umbilical cord occlusions in fetal sheep," Journal of the Society for Gynecologic Investigation, vol. 11, no. 7, pp. 449-456, 2004.

[9] H. Gonzalez, C. J. Hunter, L. Bennet, G. G. Power, and A. J. Gunn, "Cerebral oxygenation during postasphyxial seizures in near-term fetal sheep," Journal of Cerebral Blood Flow and Metabolism, vol. 25, no. 7, pp. 911-918, 2005. 
[10] S. R. Mayoral, G. Omar, and A. A. Penn, "Sex differences in a hypoxia model of preterm brain damage," Pediatric Research, vol. 66 , no. 3, pp. 248-253, 2009.

[11] M. L. Schwartz, F. Vaccarino, M. Chacon, W. Li Yan, L. R. Ment, and W. B. Stewart, "Chronic neonatal hypoxia leads to long term decreases in the volume and cell number of the rat cerebral cortex," Seminars in Perinatology, vol. 28, no. 6, pp. 379-388, 2004.

[12] R. D. Barrett, L. Bennet, J. Davidson et al., "Destruction and reconstruction: hypoxia and the developing brain," Birth Defects Research Part C-Embryo Today, vol. 81, no. 3, pp. 163176, 2007.

[13] A. J. Gunn and L. Bennet, "Fetal hypoxia insults and patterns of brain injury: insights from animal models," Clinics in Perinatology, vol. 36, no. 3, pp. 579-593, 2009.

[14] M. van Handel, H. Swaab, L. S. De Vries, and M. J. Jongmans, "Long-term cognitive and behavioral consequences of neonatal encephalopathy following perinatal asphyxia: a review," European Journal of Pediatrics, vol. 166, no. 7, pp. 645-654, 2007.

[15] J. C. Silbereis, E. J. Huang, S. A. Back, and D. H. Rowitch, "Towards improved animal models of neonatal white matter injury associated with cerebral palsy," Disease Models and Mechanisms, vol. 3, no. 11-12, pp. 678-688, 2010.

[16] L. T. L. Sie, M. S. van der Knaap, J. Oosting, L. S. De Vries, H. N. Lafeber, and J. Valk, "MR patterns of hypoxic-ischemic brain damage after prenatal, perinatal or postnatal asphyxia," Neuropediatrics, vol. 31, no. 3, pp. 128-136, 2000.

[17] C. McLean and D. Ferriero, "Mechanisms of hypoxic-ischemic injury in the term infant," Seminars in Perinatology, vol. 28, no. 6, pp. 425-432, 2004.

[18] F. J. Northington, "Brief update on animal models of hypoxicischemic encephalopathy and neonatal stroke," ILAR Journal, vol. 47, no. 1, pp. 32-38, 2006.

[19] S. Fotopoulos, K. Pavlou, H. Skouteli, I. Papassotiriou, N. Lipsou, and M. Xanthou, "Early markers of brain damage in premature low-birth-weight neonates who suffered from perinatal asphyxia and/or infection," Biology of the Neonate, vol. 79, no. 3-4, pp. 213-218, 2001.

[20] M. Allin, H. Matsumoto, A. M. Santhouse et al., "Cognitive and motor function and the size of the cerebellum in adolescents born very pre-term," Brain, vol. 124, part 1, pp. 60-66, 2001.

[21] E. B. Isaacs, C. J. Edmonds, W. K. Chong, A. Lucas, R. Morley, and D. G. Gadian, "Brain morphometry and IQ measurements in preterm children," Brain, vol. 127, part 12, pp. 2595-2607, 2004.

[22] O. G. Casiro, D. M. Moddemann, R. S. Stanwick, V. K. Panikkar-Thiessen, H. Cowan, and M. S. Cheang, "Language development of very low birth weight infants and fullterm controls at 12 months of age," Early Human Development, vol. 24, no. 1, pp. 65-77, 1990.

[23] N. Marlow, A. S. Rose, C. E. Rands, and E. S. Draper, "Neuropsychological and educational problems at school age associated with neonatal encephalopathy," Archives of Disease in Childhood: Fetal and Neonatal Edition, vol. 90, no. 5, pp. F380-F387, 2005.

[24] K. J. Steinman, M. L. Gorno-Tempini, D. V. Glidden et al., "Neonatal watershed brain injury on magnetic resonance imaging correlates with verbal IQ at 4 years," Pediatrics, vol. 123, no. 3, pp. 1025-1030, 2009.

[25] J. A. Tioseco, H. Aly, J. Essers, K. Patel, and A. A. E. El-Mohandes, "Male sex and intraventricular hemorrhage," Pediatric Critical Care Medicine, vol. 7, no. 1, pp. 40-44, 2006.
[26] J. Donders and N. M. Hoffman, "Gender differences in learning and memory after pediatric traumatic brain injury," Neuropsychology, vol. 16, no. 4, pp. 491-499, 2002.

[27] M. D. Lauterbach, S. Raz, and C. J. Sander, "Neonatal hypoxic risk in preterm birth infants: the influence of sex and severity of respiratory distress on cognitive recovery," Neuropsychology, vol. 15, no. 3, pp. 411-420, 2001.

[28] S. Raz, A. K. Debastos, J. B. Newman, and D. Batton, "Extreme prematurity and neuropsychological outcome in the preschool years," Journal of the International Neuropsychological Society, vol. 16, no. 1, pp. 169-179, 2010.

[29] K. Costeloe, E. Hennessy, A. T. Gibson, N. Marlow, and A. R. Wilkinson, "The EPICure study: outcomes to discharge from hospital for infants born at the threshold of viability," Pediatrics, vol. 106, no. 4 I, pp. 659-671, 2000.

[30] T. Gualtieri and R. E. Hicks, "An immunoreactive theory of selective male affliction," Behavioral and Brain Sciences, vol. 8, pp. 427-441, 1985.

[31] S. R. Kesler, L. R. Ment, B. Vohr et al., "Volumetric analysis of regional cerebral development in preterm children," Pediatric Neurology, vol. 31, no. 5, pp. 318-325, 2004.

[32] M. Rutter, A. Caspi, and T. E. Moffitt, "Using sex differences in psychopathology to study causal mechanisms: unifying issues and research strategies," Journal of Child Psychology and Psychiatry and Allied Disciplines, vol. 44, no. 8, pp. 1092-1115, 2003.

[33] S. Raz, M. D. Lauterbach, T. L. Hopkins et al., "A female advantage in cognitive recovery from early cerebral insult," Developmental Psychology, vol. 31, no. 6, pp. 958-966, 1995.

[34] M. Hines, "Early androgen influences on human neural and behavioural development," Early Human Development, vol. 84, no. 12 , pp. 805-807, 2008.

[35] R. Christine Knickmeyer and S. Baron-Cohen, "Fetal testosterone and sex differences," Early Human Development, vol. 82, no. 12, pp. 755-760, 2006.

[36] M. M. McCarthy, "Estradiol and the developing brain," Physiological Reviews, vol. 88, no. 1, pp. 91-124, 2008.

[37] S. H. Yang, E. Perez, J. Cutright et al., “Testosterone increases neurotoxicity of glutamate in vitro and ischemia-reperfusion injury in an animal model," Journal of Applied Physiology, vol. 92, no. 1, pp. 195-201, 2002.

[38] L. Du, H. Bayir, Y. Lai et al., "Innate gender-based proclivity in response to cytotoxicity and programmed cell death pathway," Journal of Biological Chemistry, vol. 279, no. 37, pp. 3856338570, 2004.

[39] G. D. Rosen, A. E. Herman, and A. M. Galaburda, "Sex differences in the effects of early neocortical injury on neuronal size distribution of the medial geniculate nucleus in the rat are mediated by perinatal gonadal steroids," Cerebral Cortex, vol. 9, no. 1, pp. 27-34, 1999.

[40] C. A. Hill, S. W. Threlkeld, and R. H. Fitch, "Early testosterone modulated sex differences in behavioral outcome following neonatal hypoxia ischemia in rats," International Journal of Developmental Neuroscience, vol. 29, no. 4, pp. 381-388, 2011.

[41] J. T. Lang and L. D. McCullough, "Pathways to ischemic neuronal cell death: are sex differences relevant?" Journal of Translational Medicine, vol. 6, article 33, 2008.

[42] S. Renolleau, S. Fau, and C. Charriaut-Marlangue, "Genderrelated differences in apoptotic pathways after neonatal cerebral ischemia," Neuroscientist, vol. 14, no. 1, pp. 46-52, 2008.

[43] C. Zhu, F. Xu, X. Wang et al., "Different apoptotic mechanisms are activated in male and female brains after neonatal hypoxiaischaemia," Journal of Neurochemistry, vol. 96, no. 4, pp. 10161027, 2006. 
[44] H. Hagberg, M. A. Wilson, H. Matsushita et al., "PARP-1 gene disruption in mice preferentially protects males from perinatal brain injury," Journal of Neurochemistry, vol. 90, no. 5, pp. 1068-1075, 2004.

[45] C. H. A. Nijboer, F. Groenendaal, A. Kavelaars, H. H. Hagberg, F. Van Bel, and C. J. Heijnen, "Gender-specific neuroprotection by 2 -iminobiotin after hypoxia-ischemia in the neonatal rat via a nitric oxide independent pathway," Journal of Cerebral Blood Flow and Metabolism, vol. 27, no. 2, pp. 282-292, 2007.

[46] S. Renolleau, S. Fau, C. Goyenvalle et al., "Specific caspase inhibitor Q-VD-OPh prevents neonatal stroke in P7 rat: a role for gender," Journal of Neurochemistry, vol. 100, no. 4, pp. 1062-1071, 2007.

[47] Q. L. Deveraux and J. C. Reed, "IAP family proteins-suppressors of apoptosis," Genes and Development, vol. 13, no. 3, pp. 239-252, 1999.

[48] Q. L. Deveraux, R. Takahashi, G. S. Salvesen, and J. C. Reed, "X-linked IAP is a direct inhibitor of cell-death proteases," Nature, vol. 388, no. 6639, pp. 300-304, 1997.

[49] Q. L. Deveraux, N. Roy, H. R. Stennicke et al., "IAPs block apoptotic events induced by caspase- 8 and cytochrome $\mathrm{c}$ by direct inhibition of distinct caspases," EMBO Journal, vol. 17, no. 8, pp. 2215-2223, 1998.

[50] I. Ferrer and A. M. Planas, "Signaling of cell death and cell survival following focal cerebral ischemia: life and death struggle in the penumbra," Journal of Neuropathology and Experimental Neurology, vol. 62, no. 4, pp. 329-339, 2003.

[51] S. M. Srinivasula and J. D. Ashwell, "IAPs: what's in a name?" Molecular Cell, vol. 30, no. 2, pp. 123-135, 2008.

[52] L. Carrel and H. F. Willard, "X-inactivation profile reveals extensive variability in X-linked gene expression in females," Nature, vol. 434, no. 7031, pp. 400-404, 2005.

[53] M. M. McCarthy, "Molecular aspects of sexual differentiation of the rodent brain," Psychoneuroendocrinology, vol. 19, no. 57, pp. 415-427, 1994.

[54] R. H. Fitch and V. H. Denenberg, "A role for ovarian hormones in sexual differentiation of the brain," Behavioral and Brain Sciences, vol. 21, no. 3, pp. 311-352, 1998.

[55] A. P. Arnold, E. F. Rissman, and G. J. De Vries, "Two perspectives on the origin of sex differences in the brain," Annals of the New York Academy of Sciences, vol. 1007, pp. 176188, 2003.

[56] Y. Sakuma, "Gonadal steroid action and brain sex differentiation in the rat," Journal of Neuroendocrinology, vol. 21, no. 4, pp. 410-414, 2009.

[57] A. P. Arnold, "Sex chromosomes and brain gender," Nature Reviews Neuroscience, vol. 5, no. 9, pp. 701-708, 2004.

[58] A. P. Arnold and P. S. Burgoyne, "Are XX and XY brain cells intrinsically different?" Trends in Endocrinology and Metabolism, vol. 15, no. 1, pp. 6-11, 2004.

[59] A. P. Arnold, J. Xu, W. Grisham, X. Chen, Y. H. Kim, and Y. Itoh, "Minireview: sex chromosomes and brain sexual differentiation," Endocrinology, vol. 145, no. 3, pp. 1057-1062, 2004.

[60] N. J. MacLusky, A. Philips, C. Hurlburt, and F. Naftolin, "Estrogen formation in the developing rat brain: sex differences in aromatase activity during early post-natal life," Psychoneuroendocrinology, vol. 10, no. 3, pp. 355-361, 1985.

[61] J. Weisz and I. L. Ward, "Plasma testosterone and progesterone titers of pregnant rats, their male and female fetuses, and neonatal offspring," Endocrinology, vol. 106, no. 1, pp. 306316, 1980.

[62] R. P. Michael, R. W. Bonsall, and H. D. Rees, "Sites at which testosterone may act as an estrogen in the brain of the male primate," Neuroendocrinology, vol. 46, no. 6, pp. 511-521, 1987.

[63] M. Goto, K. P. Hanley, J. Marcos et al., "In humans, early cortisol biosynthesis provides a mechanism to safeguard female sexual development," Journal of Clinical Investigation, vol. 116, no. 4, pp. 953-960, 2006.

[64] A. T. M. Konkle and M. M. McCarthy, "Developmental time course of estradiol, testosterone, and dihydrotestosterone levels in discrete regions of male and female rat brain," Endocrinology, vol. 152, no. 1, pp. 223-235, 2011.

[65] J. L. Nuñez and M. M. McCarthy, "Androgens predispose males to GABAA-mediated excitotoxicity in the developing hippocampus," Experimental Neurology, vol. 210, no. 2, pp. 699-708, 2008.

[66] T. Hawk, Y. Q. Zhang, G. Rajakumar, A. L. Day, and J. W. Simpkins, "Testosterone increases and estradiol decreases middle cerebral artery occlusion lesion size in male rats," Brain Research, vol. 796, no. 1-2, pp. 296-298, 1998.

[67] J. Cheng, N. J. Alkayed, and P. D. Hurn, "Deleterious effects of dihydrotestosterone on cerebral ischemic injury," Journal of Cerebral Blood Flow and Metabolism, vol. 27, no. 9, pp. 15531562, 2007.

[68] O. Elwan, M. Abdallah, I. Issa, Y. Taher, and M. El-Tamawy, "Hormonal changes in cerebral infarction in the young and elderly," Journal of the Neurological Sciences, vol. 98, no. 2-3, pp. 235-243, 1990.

[69] L. L. Jeppesen, H. S. Jørgensen, H. Nakayama, H. O. Raaschou, T. S. Olsen, and K. Winther, "Decreased serum testosterone in men with acute ischemic stroke," Arteriosclerosis, Thrombosis, and Vascular Biology, vol. 16, no. 6, pp. 749-754, 1996.

[70] J. Cheng, W. Hu, T. J. Toung et al., "Age-dependent effects of testosterone in experimental stroke," Journal of Cerebral Blood Flow and Metabolism, vol. 29, no. 3, pp. 486-494, 2009.

[71] W. C. J. Lan, M. Priestley, S. R. Mayoral, L. Tian, M. Shamloo, and A. A. Penn, "Sex-specific cognitive deficits and regional brain volume loss in mice exposed to chronic, sublethal hypoxia," Pediatric Research, vol. 70, no. 1, pp. 15-20, 2011.

[72] C. A. Hill, S. W. Threlkeld, G. D. Rosen, and R. H. Fitch, "Sex differences in rapid auditory processing deficits associated with neonatal hypoxia-ischemia in rats," 2007, Society for Neuroscience.

[73] C. A. Hill, M. L. Alexander, L. D. McCullough, and R. H. Fitch, "Inhibition of X-linkedinhibitor of apoptosis with embelin differentially affects male versus female behavioral outcome following neonatal hypoxia-ischemia in rats," Developmental Neuroscience. In press.

[74] S. R. Mayoral, G. Omar, and A. A. Penn, "Sex differences in a hypoxia model of preterm brain damage," Pediatric Research, vol. 66, no. 3, pp. 248-253, 2009.

[75] L. D. McCullough and P. D. Hurn, "Estrogen and ischemic neuroprotection: an integrated view," Trends in Endocrinology and Metabolism, vol. 14, no. 5, pp. 228-235, 2003.

[76] L. C. Turtzo and L. D. McCullough, "Sex differences in stroke," Cerebrovascular Diseases, vol. 26, no. 5, pp. 462-474, 2008.

[77] H. V. O. Carswell, N. H. Anderson, J. J. Morton, J. McCulloch, A. F. Dominiczak, and I. M. Macrae, "Investigation of estrogen status and increased stroke sensitivity on cerebral blood flow after a focal ischemic insult," Journal of Cerebral Blood Flow and Metabolism, vol. 20, no. 6, pp. 931-936, 2000.

[78] Y. Yamori, R. Horie, M. Sato, and K. Ohta, "Prophylactic trials for stroke in stroke prone SHR. Effect of sex hormones," Japanese Heart Journal, vol. 17, no. 3, pp. 404-406, 1976. 
[79] P. S. Herson, I. P. Koerner, and P. D. Hurn, "Sex, sex steroids, and brain injury," Seminars in Reproductive Medicine, vol. 27, no. 3, pp. 229-239, 2009.

[80] F. Liu, Z. Li, J. Li, C. Siegel, R. Yuan, and L. D. Mccullough, "Sex differences in caspase activation after stroke," Stroke, vol. 40, no. 5, pp. 1842-1848, 2009.

[81] R. H. Fitch, C. P. Brown, P. Tallal, and G. D. Rosen, "Effects of sex and MK-801 on auditory-processing deficits associated with developmental microgyric lesions in rats," Behavioral Neuroscience, vol. 111, no. 2, pp. 404-412, 1997.

[82] A. M. Peiffer, G. D. Rosen, and R. H. Fitch, "Sex differences in rapid auditory processing deficits in microgyric rats," Developmental Brain Research, vol. 148, no. 1, pp. 53-57, 2004.

[83] "Introduction to apoptosis," 2003, http://www.celldeath.de/ encyclo/aporev/apointro.pdf.

[84] S. S. Billiards, C. R. Pierson, R. L. Haynes, R. D. Folkerth, and H. C. Kinney, "Is the late preterm infant more vulnerable to gray matter injury than the term infant?" Clinics in Perinatology, vol. 33, no. 4, pp. 915-933, 2006.

[85] M. V. Johnston, A. Ishida, W. N. Ishida, H. B. Matsushita, A. Nishimura, and M. Tsuji, "Plasticity and injury in the developing brain," Brain and Development, vol. 31, no. 1, pp. 110, 2009.

[86] M. Yuan, C. Siegel, Z. Zeng, J. Li, F. Liu, and L. D. McCullough, "Sex differences in the response to activation of the poly (ADP-ribose) polymerase pathway after experimental stroke," Experimental Neurology, vol. 217, no. 1, pp. 210-218, 2009.

[87] B. B. Cho and L. H. Toledo-Pereyra, "Caspase-independent programmed cell death following ischemic stroke," Journal of Investigative Surgery, vol. 21, no. 3, pp. 141-147, 2008.

[88] J. G. Mabley, E. M. Horváth, K. G. K. Murthy et al., "Gender differences in the endotoxin-induced inflammatory and vascular responses: potential role of poly(ADP-ribose) polymerase activation," Journal of Pharmacology and Experimental Therapeutics, vol. 315, no. 2, pp. 812-820, 2005.

[89] L. D. McCullough, Z. Zeng, K. K. Blizzard, I. Debchoudhury, and P. D. Hurn, "Ischemic nitric oxide and poly (ADP-ribose) polymerase-1 in cerebral ischemia: male toxicity, female protection," Journal of Cerebral Blood Flow and Metabolism, vol. 25, no. 4, pp. 502-512, 2005.

[90] A. Chiarugi, "Poly(ADP-ribosyl)ation and stroke," Pharmacological Research, vol. 52, no. 1, pp. 15-24, 2005.

[91] Z. Nikolovska-Coleska, L. Xu, Z. Hu et al., "Discovery of embelin as a cell-permeable, small-molecular weight inhibitor of XIAP through structure-based computational screening of a traditional herbal medicine three-dimensional structure database," Journal of Medicinal Chemistry, vol. 47, no. 10, pp. 2430-2440, 2004.

[92] R. Askalan, R. Salweski, U. I. Tuor, J. Hutchison, and C. Hawkins, "X-linked inhibitor of apoptosis protein expression after ischemic injury in the human and rat developing brain," Pediatric Research, vol. 65, no. 1, pp. 21-26, 2009.

[93] J. C. Russell, H. Whiting, N. Szuflita, and M. A. Hossain, "Nuclear translocation of X-linked inhibitor of apoptosis (XIAP) determines cell fate after hypoxia ischemia in neonatal brain," Journal of Neurochemistry, vol. 106, no. 3, pp. 13571370, 2008.

[94] X. Wang, C. Zhu, X. Wang et al., "X-linked inhibitor of apoptosis (XIAP) protein protects against caspase activation and tissue loss after neonatal hypoxia-ischemia," Neurobiology of Disease, vol. 16, no. 1, pp. 179-189, 2004.

[95] B. Vischioni, P. van der Valk, S. W. Span, F. A. E. Kruyt, J. A. Rodriguez, and G. Giaccone, "Expression and localization of inhibitor of apoptosis proteins in normal human tissues," Human Pathology, vol. 37, no. 1, pp. 78-86, 2006.

[96] H. Harlin, S. Birkey Reffey, C. S. Duckett, T. Lindsten, and C. B. Thompson, "Characterization of XIAP-deficient mice," Molecular and Cellular Biology, vol. 21, no. 10, pp. 3604-3608, 2001.

[97] A. P. Arnold and X. Chen, "What does the "four core genotypes" mouse model tell us about sex differences in the brain and other tissues?" Frontiers in Neuroendocrinology, vol. 30, no. 1, pp. 1-9, 2009. 


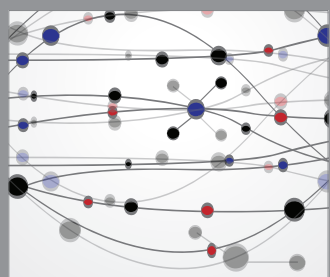

The Scientific World Journal
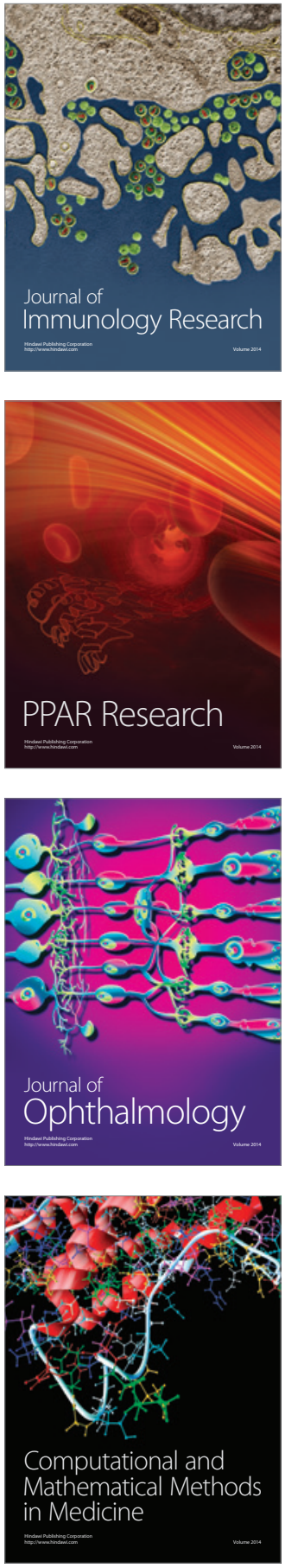

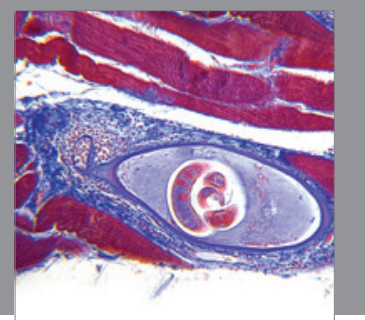

Gastroenterology

Research and Practice
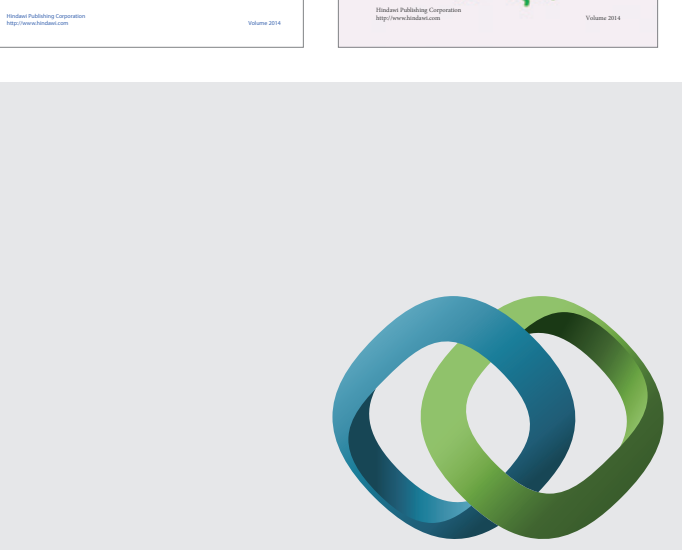

\section{Hindawi}

Submit your manuscripts at

http://www.hindawi.com
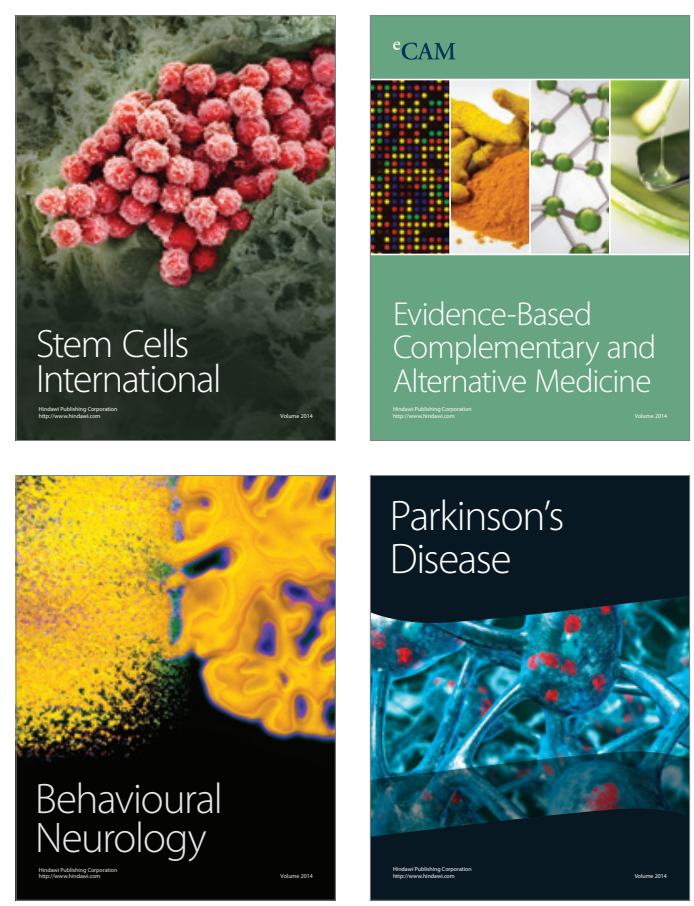

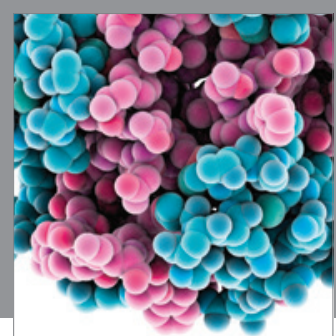

Journal of
Diabetes Research

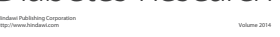

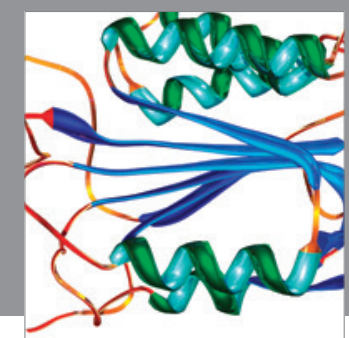

Disease Markers
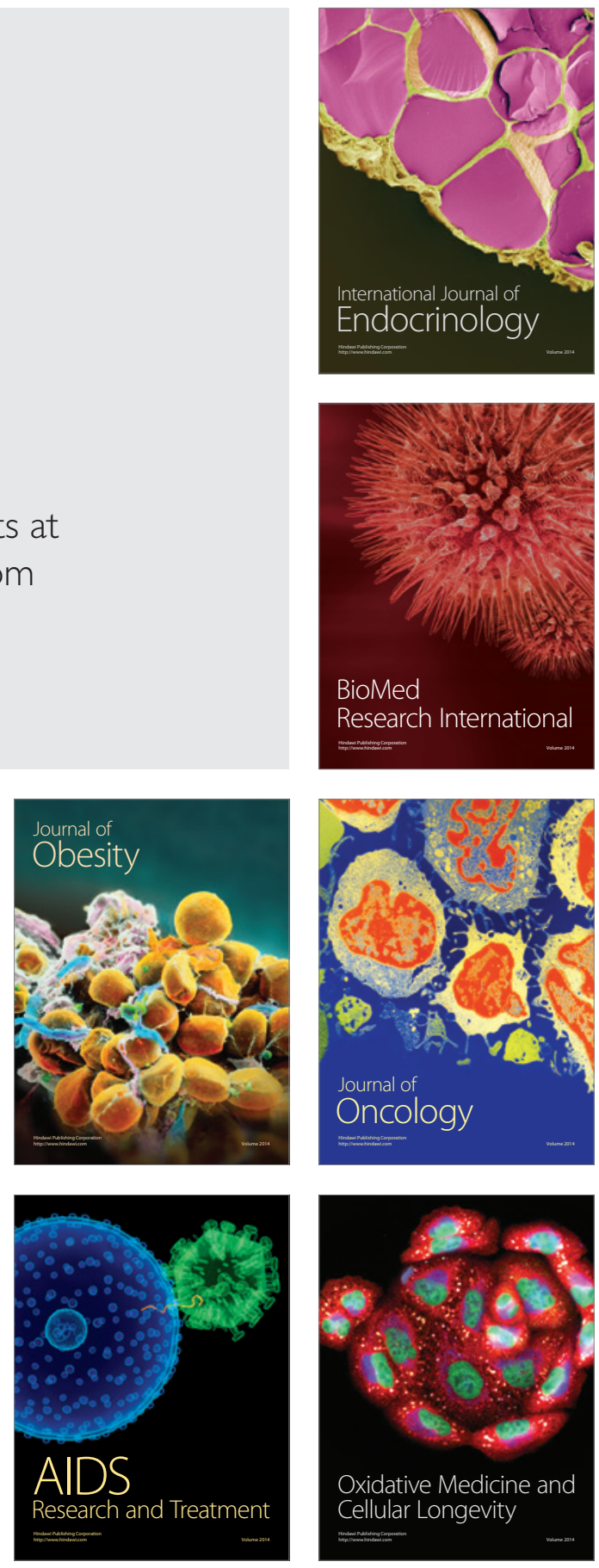